\title{
UNIQUENESS VIA PROBABILISTIC INTERPRETATION FOR THE DISCRETE COAGULATION FRAGMENTATION EQUATION
}

\author{
BENJAMIN JOURDAIN *
}

\begin{abstract}
In this paper, supposing that either the initial data is small or the fragmentation phenomenon dominates the coagulation, we associate a nonlinear stochastic process with any solution of the mass-flow equation obtained from the discrete Smoluchowski coagulation fragmentation equation by a natural change of variables. This enables us to deduce uniqueness for the mass flow equation and therefore for the corresponding Smoluchowski equation thanks to a coupling argument.
\end{abstract}

\section{Introduction}

The discrete Smoluchowski coagulation fragmentation equation describes the evolution of the expected number $c_{t}(i)$ of clusters with mass $i \in \mathbb{N}^{*}$ when two clusters with respective masses $i$ and $j$ coagulate at rate $K_{i, j}=K_{j, i}$ to form a cluster with mass $i+j$ whereas a cluster with mass $i$ breaks up at rate $F_{j, i-j}=F_{i-j, j}$ when $j \neq i / 2$ and $\frac{1}{2} F_{\frac{i}{2}}, \frac{i}{2}$ otherwise into two clusters with masses $j$ and $i-j$ :

$$
\begin{aligned}
\dot{c}_{t}(i)= & \frac{1}{2} \sum_{j=1}^{i-1}\left(K_{j, i-j} c_{t}(j) c_{t}(i-j)-F_{j, i-j} c_{t}(i)\right) \\
& -\sum_{j \in \mathbb{N}^{*}}\left(K_{i, j} c_{t}(i) c_{t}(j)-F_{i, j} c_{t}(i+j)\right) .
\end{aligned}
$$

Throughout this paper, we assume that

$$
\exists \kappa>0, \forall i, j \in \mathbb{N}^{*}, K_{i, j} \leq \kappa i j \text { and } c_{0} \in \mathbb{R}_{+}^{\mathbb{N}^{*}} \text { satisfies } \sum_{i \in \mathbb{N}^{*}} i c_{0}(i)=1 \text {. }
$$

Since both in the coagulation phenomenon and the reverse fragmentation reaction, the mass is preserved contrary to the number of clusters, in order to give a probabilistic interpretation, we are interested in the evolution of $p_{t}(i)=i c_{t}(i)$. Multiplying (1.1) by $i$ and using the symmetry of both kernels $K$ and $F$, we obtain the so-called mass-flow equation satisfied by the new variables $p_{t}(i)$ :

$$
\begin{aligned}
p_{0}(i)=i c_{0}(i), \quad \dot{p}_{t}(i)= & \sum_{j=1}^{i-1}\left(\tilde{K}_{j, i-j} p_{t}(j) p_{t}(i-j)-\tilde{F}_{j, i-j} p_{t}(i)\right) \\
& -\sum_{j \in \mathbb{N}^{*}}\left(\tilde{K}_{i, j} p_{t}(i) p_{t}(j)-\tilde{F}_{i, j} p_{t}(i+j)\right)
\end{aligned}
$$

with modified kernels

$$
\tilde{K}_{i, j}=\frac{1}{j} K_{i, j} \text { and } \tilde{F}_{i, j}=\frac{i}{i+j} F_{i, j}
$$

In this paper we want to associate with a given solution $p_{t}$ of this equation a stochastic process $t \rightarrow X_{t}$ such that $\forall i \in \mathbb{N}^{*}, \mathbb{P}\left(X_{t}=i\right)=p_{t}(i)$. Our final goal is to prove uniqueness for (1.3) and therefore for (1.1) without upper-bounding the fragmentation

*ENPC-CERMICS, 6-8 av Blaise Pascal, Cité Descartes, Champs sur Marne, 77455 Marne-laVallée Cedex 2, France (jourdain@cermics.enpc.fr), http://cermics.enpc.fr/ jourdain/home.html 
kernel $F$. In the pure fragmentation case $(K \equiv 0)$, it is then possible to construct solutions of (1.1) with increasing total mass $\sum_{i \in \mathbb{N}^{*}} i c_{t}(i)=\sum_{i \in \mathbb{N}^{*}} p_{t}(i)$ [BC]. These solutions have to be rejected for obvious physical reasons. In the pure coagulation case $(F \equiv 0)$, it may happen that the total mass decreases after a finite time. Intuitively, this phenomenon called gelation corresponds to the formation of an infinite cluster. That is why we are only going to consider solutions with non-increasing total mass.

Definition 1.1. Let $T \in(0,+\infty]$. We say that $t \in[0, T) \rightarrow p_{t} \in \mathbb{R}_{+}^{\mathbb{N}^{*}}$ solves on $[0, T)$ if

1. $t \rightarrow \sum_{i \in \mathbb{N}^{*}} p_{t}(i)$ is non-increasing

2. $\forall t \in[0, T), \forall i \in \mathbb{N}^{*}, s \rightarrow \sum_{j \in \mathbb{N}^{*}} \tilde{F}_{i, j} p_{s}(i+j)$ is integrable on $(0, t)$ and

$$
\begin{aligned}
p_{t}(i)=p_{0}(i)+\int_{0}^{t} & \sum_{j=1}^{i-1}\left(\tilde{K}_{j, i-j} p_{s}(j) p_{s}(i-j)-\tilde{F}_{j, i-j} p_{s}(i)\right) \\
& -\sum_{j \in \mathbb{N}^{*}}\left(\tilde{K}_{i, j} p_{s}(j) p_{s}(i)-\tilde{F}_{i, j} p_{s}(i+j)\right) d s .
\end{aligned}
$$

REMARK 1.2. If $p$ satisfies the previous definition, according to 1. and (1.2), $\sum_{j \in \mathbb{N}^{*}} \tilde{K}_{i, j} p_{s}(i) p_{s}(j)$ is smaller than $\kappa i$ and thus locally integrable w.r.t. s.

The first section of the paper is dedicated to the probabilistic interpretation of solutions of (1.3). In the second section, we deduce results concerning the dependence of solutions of this equation w.r.t. the initial condition $p_{0}$.

Notations. Let $\overline{\mathbb{N}}=\mathbb{N}^{*} \cup\{\infty\}$ endowed with the discrete topology and $\mathcal{P}(\overline{\mathbb{N}})$ (resp. $\mathcal{P}(\overline{\mathbb{N}} \times \overline{\mathbb{N}}))$ denote the set of probability measures on $\overline{\mathbb{N}}($ resp $\overline{\mathbb{N}} \times \overline{\mathbb{N}})$ endowed with the total variation metric (which metrizes the narrow convergence topology).

For $(p, q) \in \mathcal{P}(\overline{\mathbb{N}})^{2}$ the subset of $\mathcal{P}(\overline{\mathbb{N}} \times \overline{\mathbb{N}})$ consisting in probability measures with respective marginals $p$ and $q$ is denoted by $\mathcal{P}_{p, q}$.

If $p \in \mathbb{R}_{+}^{\mathbb{N}^{*}}$ is such that $\sum_{i \in \mathbb{N}^{*}} p(i) \leq 1$, we extend $p$ to $\mathcal{P}(\overline{\mathbb{N}})$ by setting $p(\infty)=$ $1-\sum_{i \in \mathbb{N}^{*}} p(i)$.

\section{Nonlinear processes associated with (1.3)}

Let $p_{t}$ solve $(1.3)$ on $[0, T)$. In this section, we want to construct a stochastic process $t \rightarrow X_{t} \in \overline{\mathbb{N}}$ such that $\forall i \in \overline{\mathbb{N}}, \mathbb{P}\left(X_{t}=i\right)=p_{t}(i)$. We first remark that $p_{t}$ solves the linear equation

$$
\begin{aligned}
r_{0}=p_{0}, \quad \dot{r}_{t}(i)= & \sum_{j=1}^{i-1}\left(\tilde{K}_{j, i-j} r_{t}(j) p_{t}(i-j)-\tilde{F}_{j, i-j} r_{t}(i)\right) \\
& -\sum_{j \in \mathbb{N}^{*}}\left(\tilde{K}_{i, j} r_{t}(i) p_{t}(j)-\tilde{F}_{i, j} r_{t}(i+j)\right) .
\end{aligned}
$$

This linear equation is the Fokker-Planck (or Kolmogorov forward) equation associated with a jump process. More precisely, let $\left(X_{t}\right)_{t \in[0, T)}$ denote a $\overline{\mathbb{N}}$-valued jump process with transition rates

$$
\lambda_{p}(t, i, j)=1_{\{i<\infty\}}\left(1_{\{1 \leq j \leq i-1\}} \tilde{F}_{j, i-j}+1_{\{i<j<\infty\}} \tilde{K}_{i, j-i} p_{t}(j-i)\right)
$$

starting from $X_{0}$ distributed according to $p_{0}$. The intuitive meaning of the transition rates is the following: when $X_{t}$ is equal to $i$ the probability for a jump leading from $i$ 
to $j$ to occur on $[t, t+\Delta t]$ is equal to $\lambda_{p}(t, i, j) \Delta t+o(\Delta t)$. Denoting $q_{t}(i)=\mathbb{P}\left(X_{t}=i\right)$, one has

$$
\begin{aligned}
q_{t+\Delta t}(i) & =\mathbb{P}\left(X_{t}=i \text { and no jump on }[t, t+\Delta t]\right) \\
& +\mathbb{P}\left(X_{t} \neq i \text { and a jump leading to } i \text { on }[t, t+\Delta t]\right) \\
& +\mathbb{P}(\text { at least two jumps on }[t, t+\Delta t]) \\
& =q_{t}(i)\left(1-\sum_{j \neq i} \lambda_{p}(t, i, j) \Delta t\right)+\sum_{j \neq i} q_{t}(j) \lambda_{p}(t, j, i) \Delta t+o(\Delta t) .
\end{aligned}
$$

Subtracting $q_{t}(i)$ to both sides, dividing by $\Delta t$ and letting $\Delta t \rightarrow 0$, one obtains formally that $q_{t}(i)=\mathbb{P}\left(X_{t}=i\right)$ solves $(2.1)$.

At this stage, the dynamics of the process $X_{t}$ is not entirely specified. Indeed, the successive jump times $\left(\tau_{n}\right)_{n \geq 1}$ may accumulate before $T$ and we need to make precise what happens after. In case of such an accumulation, necessarily $\lim _{n \rightarrow+\infty} X_{\tau_{n}}=\infty$ and we set $X_{t}=\infty$ on $\left[\lim _{n} \tau_{n}, T\right)$. With this choice, one may check rigorously [J] that $q_{t}(i)=\mathbb{P}\left(X_{t}=i\right)$ is a solution of (2.1) such that $t \rightarrow \sum_{i \in \mathbb{N}^{*}} q_{t}(i)$ is non-increasing.

Hence it is enough to prove uniqueness of such solutions to obtain the desired probabilistic interpretation: $\forall t \in[0, T), \forall i \in \overline{\mathbb{N}}, q_{t}(i)=p_{t}(i)$. The jump process $X$ is then nonlinear in the following sense: its time-marginals $p_{t}$ appear in the definition of the transition rates.

REMARK 2.1. Without fragmentation, uniqueness for (2.1) is easily obtained by induction on $i$.

In presence of fragmentation, we are going to suppose either that the initial data $p_{0}$ is small or that the fragmentation dominates the coagulation to obtain $p_{t}=q_{t}$ thanks to the following uniqueness result

Lemma 2.2. Let $\tau \leq T$. The linear equation (2.1) admits at most one solution $t \in$ $[0, \tau) \rightarrow r_{t} \in \mathbb{R}_{+}^{\mathbb{N}^{*}}$ such that $\sum_{i \in \mathbb{N}^{*}} r_{t}(i)$ is non-increasing and $\sum_{i \in \mathbb{N}^{*}} \sup _{j \in \mathbb{N}^{*}} \tilde{K}_{i, j} r_{t}(i)$ is locally integrable w.r.t. $t$ on $[0, \tau)$. In addition, such a solution satisfies $\forall t \in$ $[0, \tau), \sum_{i \in \mathbb{N}^{*}} r_{t}(i)=1$.

Proof: Let $r_{t}$ and $\tilde{r}_{t}$ be two such solutions and $x_{t}=r_{t}-\tilde{r}_{t}$. By (2.1)

$$
\text { for } n \in \mathbb{N}^{*}, \frac{d}{d t} \sum_{i=1}^{n}\left|x_{t}(i)\right|=\sum_{i=1}^{n} s_{i}\left(\sum_{j=1}^{i-1} W_{j, i-j}-\sum_{j \in \mathbb{N}^{*}} W_{i, j}\right)
$$

with $s_{i}=\operatorname{sign}\left(x_{t}(i)\right)$ and $W_{i, j}=\tilde{K}_{i, j} x_{t}(i) p_{t}(j)-\tilde{F}_{i, j} x_{t}(i+j)$. As

$$
\sum_{i=1}^{n} s_{i} \sum_{j=1}^{i-1} W_{j, i-j}=\sum_{j=1}^{n} \sum_{i=j+1}^{n} s_{i} W_{j, i-j}=\sum_{j=1}^{n} \sum_{k=1}^{n-j} s_{j+k} W_{j, k},
$$




$$
\begin{aligned}
\frac{d}{d t} \sum_{i=1}^{n}\left|x_{t}(i)\right|= & \sum_{i=1}^{n} \sum_{j=1}^{i-1}\left(s_{i+j}-s_{i}\right)\left(\tilde{K}_{i, j} x_{t}(i) p_{t}(j)-\tilde{F}_{i, j} x_{t}(i+j)\right) \\
& +\sum_{i=1}^{n} \sum_{n+1-i \leq j<\infty} s_{i}\left(\tilde{F}_{i, j} x_{t}(i+j)-\tilde{K}_{i, j} x_{t}(i) p_{t}(j)\right) \\
\leq & \sum_{i=1}^{n} \sum_{n+1-i \leq j<\infty} \tilde{F}_{i, j}\left(r_{t}(i+j)+\tilde{r}_{t}(i+j)\right) \\
= & \frac{d}{d t} \sum_{i=1}^{n}\left(r_{t}(i)+\tilde{r}_{t}(i)\right)+\sum_{i=1}^{n} \sum_{\substack{j \geq n+1-i \\
j<\infty}} \tilde{K}_{i, j} p_{t}(j)\left(r_{t}(i)+\tilde{r}_{t}(i)\right),
\end{aligned}
$$

where the last equality follows from (2.1) and (2.2) for $s_{i}=1$ and $W_{i, j}=\tilde{K}_{i, j}\left(r_{t}(i)+\right.$ $\left.\tilde{r}_{t}(i)\right) p_{t}(j)-\tilde{F}_{i, j}\left(r_{t}(i+j)+\tilde{r}_{t}(i+j)\right)$.

Integrating the inequality on $[0, s]$ for $s<\tau$, letting $n \rightarrow+\infty$ we deduce

$$
\sum_{i \in \mathbb{N}^{*}}\left|r_{s}(i)-\tilde{r}_{s}(i)\right| \leq \limsup _{n \rightarrow+\infty} \int_{0}^{s} \sum_{i, j \in \mathbb{N}^{*}} 1_{\{i \leq n, j \geq n+1-i\}} \tilde{K}_{i, j} p_{t}(j)\left(r_{t}(i)+\tilde{r}_{t}(i)\right) d t .
$$

As $\sum_{i, j \in \mathbb{N}^{*}} \tilde{K}_{i, j} p_{t}(j)\left(r_{t}(i)+\tilde{r}_{t}(i)\right) \leq \kappa \sum_{i \in \mathbb{N}^{*}} \sup _{j \in \mathbb{N}^{*}} \tilde{K}_{i, j}\left(r_{t}(i)+\tilde{r}_{t}(i)\right)$ is integrable on $[0, s]$, we conclude by Lebesgue's theorem that $r_{s}=\tilde{r}_{s}$. Integrating the nonnegative right-hand-side of $(2.3)$ over $[0, s]$ and taking the limit $n \rightarrow+\infty$, we also obtain $\sum_{i \in \mathbb{N}^{*}} r_{t}(i) \geq \sum_{i \in \mathbb{N}^{*}} p_{0}(i)=1$.

2.1. The strong fragmentation case. We assume that there exist constants $\alpha \in(1 / 2,1], \beta>\alpha$ and $\kappa>0$ s.t.

$$
\begin{aligned}
& \forall i, j \geq 1, K_{i, j} \leq \kappa i^{\alpha} j^{\alpha} \quad \text { i.e. } \tilde{K}_{i, j} \leq \kappa i^{\alpha} j^{\alpha-1} \\
& \forall \mu \geq 0, \exists C(\mu)>0, \forall i \geq 3, \quad \sum_{j=1}^{[(i-1) / 2]} j^{\mu} F_{j, i-j} \geq C(\mu) i^{\beta+\mu} .
\end{aligned}
$$

Under this assumption denoted by (SF), existence and uniqueness for (1.1) has been proved by Da Costa [D]. Translated in terms of (1.3) his results imply the first assertion in the following proposition:

Proposition 2.3. Under (SF), (1.3) admits a unique solution $p_{t}$ on $[0,+\infty)$ and $\forall t \geq 0, \sum_{i \in \mathbb{N}^{*}} p_{t}(i)=1$. In addition, the jump process $X$ provides a probabilistic interpretation of $p_{t}: \forall t \geq 0, \forall i \in \mathbb{N}^{*}, \mathbb{P}\left(X_{t}=i\right)=p_{t}(i)$.

Since $\forall i \in \mathbb{N}^{*}, \sup _{j \in \mathbb{N}^{*}} \tilde{K}_{i, j} \leq \kappa i^{\alpha}$, combined with Lemma 2.2 the following estimate which is adapted from the proof of [D] Theorem 5.2 ensures that (1.3) has no more than one solution $r_{t}$ such that $t \rightarrow \sum_{i \in \mathbb{N}^{*}} r_{t}(i)$ is non-increasing. We easily deduce the second assertion in the Proposition.

Lemma 2.4. Under (SF), if $t \rightarrow r_{t} \in \mathbb{R}_{+}^{\mathbb{N}^{*}}$ is such that $\sum_{i \in \mathbb{N}^{*}} r_{t}(i)$ is non-increasing and solves

$$
\begin{aligned}
& r_{0}=p_{0}, \quad \dot{r}_{t}(i)=\sum_{j=1}^{i-1}\left(L_{t}^{j, i-j}-\tilde{F}_{j, i-j} r_{t}(i)\right)-\sum_{j \in \mathbb{N}^{*}}\left(L_{t}^{i, j}-\tilde{F}_{i, j} r_{t}(i+j)\right), \\
& \text { with } \forall i \in \mathbb{N}^{*}, \forall t \geq 0, L_{t}^{i, j} \geq 0 \text { for } j \in \mathbb{N}^{*} \text { and } \sum_{j \in \mathbb{N}^{*}} L_{t}^{i, j} \leq \kappa i^{\alpha} r_{t}(i)
\end{aligned}
$$


then $\forall t \geq 0, \kappa \int_{0}^{t} \sum_{i \in \mathbb{N}^{*}} i^{\alpha} r_{s}(i) d s \leq I(t)<+\infty$ with I independent of $p_{0}$.

2.2. Small initial data. Here we only suppose that the initial data is small in the following sense: $\sum_{i \in \mathbb{N}^{*}} i p_{0}(i)=\sum_{i \in \mathbb{N}^{*}} i^{2} c_{0}(i)<\infty$. By considering $n$-dimensional truncations of the infinite system (1.3), one can prove the following existence result (see $[\mathrm{J}]$ p.109):

Proposition 2.5. Let $\mathcal{T}_{p_{0}}=\left(\kappa \sum_{i \in \mathbb{N}^{*}} i p_{0}(i)\right)^{-1}$. The equation (1.3) admits a solution $p_{t}$ on $\left[0, \mathcal{T}_{p_{0}}\right)$ satisfying $\forall t \in\left[0, \mathcal{T}_{p_{0}}\right)$,

$$
\sum_{i \in \mathbb{N}^{*}} i p_{t}(i) \leq\left(\kappa\left(\mathcal{T}_{p_{0}}-t\right)\right)^{-1}
$$

From now on, we denote by $\left(w_{t}\right)_{t \in\left[0, \mathcal{T}_{p_{0}}\right)}$ such a solution for the multiplicative coagulation kernel $\kappa i j$ and in the absence of fragmentation:

$$
w_{0}=p_{0}, \dot{w}_{t}(i)=\kappa\left(\sum_{j=1}^{i-1} j w_{t}(j) w_{t}(i-j)-i w_{t}(i) \sum_{j \in \mathbb{N}^{*}} w_{t}(j)\right) .
$$

By $(2.5)$ and Lemma $2.2, \forall t \in\left[0, \mathcal{T}_{p_{0}}\right), \sum_{i \in \mathbb{N}^{*}} w_{t}(i)=1$ i.e. $w_{t}(\infty)=0$.

Since in (1.3), the coagulation kernel is smaller than $\kappa i j$ and fragmentation is possible, one intuitively feels that any solution $p_{t}$ should give less weight than $w_{t}$ to large integers and that $\sum_{i \in \mathbb{N}^{*}} i p_{t}(i)$ should be smaller than $\sum_{i \in \mathbb{N}^{*}} i w_{t}(i)$. We are going to make this intuition rigorous to prove

TheOREM 2.6. Let $p_{t}$ solve (1.3) on $[0, T)$. Then (2.5) and $\sum_{i \in \mathbb{N} *} p_{t}(i)=1$ hold on $\left[0, T \wedge \mathcal{T}_{p_{0}}\right)$. In addition the jump process $X$ provides a probabilistic interpretation of $p_{t}$ on $\left[0, T \wedge \mathcal{T}_{p_{0}}\right): \forall t \in\left[0, T \wedge \mathcal{T}_{p_{0}}\right), \forall i \in \mathbb{N}^{*}, \mathbb{P}\left(X_{t}=i\right)=p_{t}(i)$.

In order to quantify the comparison between $p_{t}$ and $w_{t}$, we introduce the following function $f$ :

LEMma 2.7. The mapping $(p, q) \in \mathcal{P}(\overline{\mathbb{N}})^{2} \rightarrow f(p, q)=\inf _{\rho \in \mathcal{P}_{p, q}} \sum_{i>j} \rho(i, j)$ is continuous and there is a measurable choice $(p, q) \in \mathcal{P}(\overline{\mathbb{N}})^{2} \rightarrow \rho_{p, q} \in \mathcal{P}(\overline{\mathbb{N}} \times \overline{\mathbb{N}})$ such that $\forall(p, q) \in \mathcal{P}(\overline{\mathbb{N}})^{2}, f(p, q)=\sum_{i>j} \rho_{p, q}(i, j)$. In addition, for $p, q, w \in \mathcal{P}(\overline{\mathbb{N}})$.

1. $(f(p, q)=0) \Leftrightarrow\left(\forall n \in \mathbb{N}^{*}, \sum_{i=1}^{n} p(i) \geq \sum_{i=1}^{n} q(i)\right) \Rightarrow(p(\infty) \leq q(\infty))$

2. If $f(p, q)=0$ and $p(\infty)=q(\infty)$ then $\sum_{i \in \mathbb{N}^{*}} i p(i) \leq \sum_{i \in \mathbb{N}^{*}} i q(i)$.

3. If $f(p, q)=0$ then $f(p, w) \leq f(q, w)$.

Proof: According to Prokhorov Theorem, for $(p, q) \in \mathcal{P}(\overline{\mathbb{N}})^{2}, \mathcal{P}_{p, q}$ is a compact subset of $\mathcal{P}(\overline{\mathbb{N}} \times \overline{\mathbb{N}})$.

Since the mapping $\rho \in \mathcal{P}(\overline{\mathbb{N}} \times \overline{\mathbb{N}}) \rightarrow \sum_{i<j} \rho(i, j)$ is Lipschitz continuous, to obtain the continuity of $f$, it is enough to prove the continuity of $(p, q) \in \mathcal{P}(\overline{\mathbb{N}})^{2} \rightarrow \mathcal{P}_{p, q}$ when the set of compact subsets of $\mathcal{P}(\overline{\mathbb{N}} \times \overline{\mathbb{N}})$ is endowed with the Prokhorov metric. This continuity property also yields the existence of a measurable choice (see for instance [SV] Chapter 12).

To prove it, the only difficulty is to check that when $\left(p_{n}, q_{n}\right)_{n}$ denotes a sequence converging to $(p, q)$ in $\mathcal{P}(\overline{\mathbb{N}})^{2}$ and $\rho \in \mathcal{P}_{p, q}$, then there exists a sequence $\rho_{n} \in \mathcal{P}_{p_{n}, q_{n}}$ converging to $\rho$ as $n \rightarrow+\infty$. Let $k \in \mathbb{N} \rightarrow\left(i_{k}, j_{k}\right) \in \overline{\mathbb{N}} \times \overline{\mathbb{N}}$ be a bijection. We define inductively for $k \in \mathbb{N}$

$$
\tilde{\rho}_{n}\left(i_{k}, j_{k}\right)=\rho\left(i_{k}, j_{k}\right) \wedge\left(p_{n}\left(i_{k}\right)-\sum_{\substack{l \leq k-1 \\ i_{l}=i_{k}}} \tilde{\rho}_{n}\left(i_{l}, j_{l}\right)\right)^{+} \wedge\left(q_{n}\left(j_{k}\right)-\sum_{\substack{l \leq k-1 \\ j_{l}=j_{k}}} \tilde{\rho}_{n}\left(i_{l}, j_{l}\right)\right)^{+}
$$


and obtain suitable probability measures by setting

$$
\rho_{n}(i, j)=\tilde{\rho}_{n}(i, j)+\frac{\left(p_{n}(i)-\sum_{k \in \overline{\mathbb{N}}} \tilde{\rho}_{n}(i, k)\right)\left(q_{n}(j)-\sum_{l \in \overline{\mathbb{N}}} \tilde{\rho}_{n}(l, j)\right)}{1-\sum_{k, l \in \overline{\mathbb{N}} \times \overline{\mathbb{N}}} \tilde{\rho}_{n}(k, l)} .
$$

Let us now give elements of proof of the numbered properties. The first one is obtained by inversion of the cumulative distribution function. We deduce the second one by remarking that for $r \in \mathcal{P}(\overline{\mathbb{N}}), \sum_{i \in \mathbb{N}^{*}} i r(i)=\sum_{n \in \mathbb{N}^{*}}\left(1-r(\infty)-\sum_{i=1}^{n-1} r(i)\right)$ and obtain the last one by considering the following probability measure on $\overline{\mathbb{N}}^{3}$ with marginals $p, q, w: 1_{\{q(j)>0\}} \frac{\rho_{p, q}(i, j) \rho_{q, w}(j, k)}{q(j)}$.

We are now ready to prove Theorem 2.6.

Proof: To conclude, it is enough to check

$$
\forall t \in\left[0, T \wedge \mathcal{T}_{p_{0}}\right), f\left(p_{t}, w_{t}\right)+f\left(q_{t}, w_{t}\right)=0 .
$$

Indeed by Lemma $2.72,(2.7)$ and $w_{t}(\infty)=0$ for $t \in\left[0, \mathcal{T}_{p_{0}}\right)$ imply

$$
\forall t \in\left[0, T \wedge \mathcal{T}_{p_{0}}\right), \max \left(\sum_{i \in \mathbb{N}^{*}} i p_{t}(i), \sum_{i \in \mathbb{N}^{*}} i q_{t}(i)\right) \leq \sum_{i \in \mathbb{N}^{*}} i w_{t}(i) \leq\left(\kappa\left(\mathcal{T}_{p_{0}}-t\right)\right)^{-1} .
$$

Combined with Lemma 2.2 , this inequality yields $\forall t \in\left[0, T \wedge \mathcal{T}_{p_{0}}\right), p_{t}=q_{t}$ and $\sum_{i \in \mathbb{N}^{*}} p_{t}(i)=1$.

In $[\mathrm{J}],(2.7)$ is obtained by comparing some solutions of linear equations and concluding by a complicated limiting procedure. Here we present a simpler and more intuitive coupling argument: let $\left(Y_{t}, \tilde{Y}_{t}\right)_{t \in\left[0, T \wedge \mathcal{T}_{p_{0}}\right)}$ denote a jump process on $\overline{\mathbb{N}}^{2}$ starting from $Y_{0}=\tilde{Y}_{0}$ distributed according $p_{0}$ and with transition rates $\lambda(t,(i, j),(k, l))$ equal to $\kappa$ times

$$
\left\{\begin{aligned}
(i \wedge j) \rho_{p_{t}, w_{t}}(k-i, l-j) \text { if } i<k<\infty \text { and } j<l<\infty \\
(i-j)^{+} p_{t}(k-i)+1_{\{j=\infty\}} i p_{t}(k-i) \text { if } i<k<\infty \text { and } j=l \\
(j-i)^{+} w_{t}(l-j)+1_{\{i<\infty\}}(i \wedge j)\left(w_{t}(l-j)-\sum_{m \in \mathbb{N}^{*}} \rho_{p_{t}, w_{t}}(m, l-j)\right) \\
+1_{\{i=\infty\}} j w_{t}(l-j) \text { if } i=k \text { and } j<l<\infty
\end{aligned}\right.
$$

and 0 otherwise. If jumps accumulate at time $s$ before $T \wedge \mathcal{T}_{p_{0}}$ then either both coordinates go to $\infty$ and remain equal to $\infty$ after or only one coordinate goes to $\infty$. In the latter case, this coordinate remains equal to $\infty$ and the other is chosen continuous at $s$ and equal to $\infty$ after a possible second accumulation of jumps.

This way $\tilde{Y}$ is a jump process with initial law $p_{0}$ and transition rates $\tilde{\lambda}(t, j, l)=$ $1_{\{j<l<+\infty\}} \kappa j w_{t}(l-j)$. By Remark 2.1, for any $t \in\left[0, T \wedge \mathcal{T}_{p_{0}}\right)$, the law of $\tilde{Y}_{t}$ is $w_{t}$. Similarly, using that $\forall t \in\left[0, T \wedge \mathcal{T}_{p_{0}}\right), w_{t}(\infty)=0$ and therefore $\forall i \in \overline{\mathbb{N}}$, $p_{t}(i)=\sum_{j \in \mathbb{N}^{*}} \rho_{p_{t}, w_{t}}(i, j)$, one checks that $Y$ is a jump process with initial law $p_{0}$ and transition rates $\lambda(t, i, k)=1_{\{i<k<+\infty\}} \kappa i p_{t}(k-i)$. Hence the law $r_{t}$ of $Y_{t}$ solves the linear equation

$$
r_{0}=p_{0}, \dot{r}_{t}(i)=\kappa\left(\sum_{j=1}^{i-1} j r_{t}(j) p_{t}(i-j)-i r_{t}(i) \sum_{j \in \mathbb{N}^{*}} p_{t}(j)\right) .
$$

According to Lemma $4.2[\mathrm{~J}]$ and Lemma 2.71 ,

$$
\forall t \in\left[0, T \wedge \mathcal{T}_{p_{0}}\right), f\left(q_{t}, r_{t}\right)=0 \text { and } f\left(p_{t}, r_{t}\right)=0
$$


As we want to prove that $\tilde{Y}_{t}$ is bigger than $Y_{t}$ the coupling $(Y, \tilde{Y})$ is constructed in order to minimize the jumps $\left(\Delta Y_{s}, \Delta \tilde{Y}_{s}\right)=\left(Y_{s}-Y_{s^{-}}, \tilde{Y}_{s}-\tilde{Y}_{s^{-}}\right)$bigger for the first coordinate than for the second. Since the law of $\left(Y_{t}, \tilde{Y}_{t}\right)$ belongs to $\mathcal{P}_{r_{t}, w_{t}}$, using the last equality and Lemma 2.73 , one obtains

$$
\begin{aligned}
f\left(p_{t}, w_{t}\right) \leq f\left(r_{t}, w_{t}\right) & \leq \mathbb{P}\left(Y_{t}>\tilde{Y}_{t}\right) \leq \mathbb{P}\left(\exists s \leq t, Y_{s^{-}} \leq \tilde{Y}_{s^{-}}<\infty \text { and } \Delta Y_{s}>\Delta \tilde{Y}_{s}\right) \\
& \leq \mathbb{E}\left(\sum_{s \leq t} 1_{\left\{Y_{s^{-}} \leq \tilde{Y}_{s^{-}}<\infty \text { and } \Delta Y_{s}>\Delta \tilde{Y}_{s}\right\}}\right) \\
& =\kappa \mathbb{E}\left(\int_{0}^{t} 1_{\left\{Y_{s^{-}} \leq \tilde{Y}_{s^{-}}<\infty\right\}} Y_{s^{-}} f\left(p_{s}, w_{s}\right) d s\right) \\
& \leq \kappa \int_{0}^{t} \sum_{i \in \mathbb{N}^{*}} i w_{s}(i) f\left(p_{s}, w_{s}\right) d s
\end{aligned}
$$

Since (2.5) holds for $w_{t}$ on $\left[0, \mathcal{T}_{p_{0}}\right)$, Gronwall's lemma implies that $\forall t \in\left[0, T \wedge \mathcal{T}_{p_{0}}\right)$, $f\left(p_{t}, w_{t}\right)=0$ and $f\left(r_{t}, w_{t}\right)=0$. We also obtain $f\left(q_{t}, w_{t}\right)=0$ by combining the last equality, (2.8) and Lemma 2.73.

3. Dependence on the initial data for (1.3)

For $p, q \in \mathcal{P}(\overline{\mathbb{N}})$, let $\|p-q\|=\sum_{i \in \overline{\mathbb{N}}}|p(i)-q(i)|$. The probabilistic interpretation of solutions of (1.3) enables us to prove the following result by a simple coupling argument.

Theorem 3.1. Let $p_{0}$ and $\tilde{p}_{0}$ be two probability measures on $\mathbb{N}^{*}$, and $p_{t}$ (resp. $\tilde{p}_{t}$ ) solve (1.3) with initial data $p_{0}$ (resp. $\left.\tilde{p}_{0}\right)$ on $[0, T)$ (resp. $[0, \tilde{T})$ ).

1. Under (SF), $\forall t \in[0, T \wedge \tilde{T}),\left\|p_{t}-\tilde{p}_{t}\right\| \leq\left\|p_{0}-\tilde{p}_{0}\right\| e^{I(t)}$ where $I(t)$ is the bound given in Lemma 2.4 .

2. If $\sum_{i \in \mathbb{N}^{*}} i \tilde{p}_{0}(i) \leq \sum_{i \in \mathbb{N}^{*}} i p_{0}(i)<+\infty$, then for $\mathcal{T}_{p}=\left(\kappa \sum_{i \in \mathbb{N}^{*}} i p(i)\right)^{-1}$,

$$
\forall t \in\left[0, T \wedge \tilde{T} \wedge \mathcal{T}_{p_{0}}\right),\left\|p_{t}-\tilde{p}_{t}\right\| \leq \frac{\mathcal{T}_{\tilde{p}_{0}}\left\|p_{0}-\tilde{p}_{0}\right\|}{\mathcal{T}_{\tilde{p}_{0}}-t} .
$$

For the choice $\tilde{p}_{0}=p_{0}$, we easily deduce:

Corollary 3.2. Uniqueness holds for (1.3) on the time interval $\left[0, \mathcal{T}_{p_{0}}\right)$ if $\sum_{i \in \mathbb{N}^{*}} i p_{0}(i)$ $<+\infty$ and on $[0,+\infty)$ under $(\mathbf{S F})$.

Proof of Theorem 3.1: For $(p, q) \in \mathcal{P}(\overline{\mathbb{N}})^{2}$, let us define $\bar{\rho} \in \mathcal{P}_{p, q}$ by

$$
\forall(i, j) \in \overline{\mathbb{N}}^{2}, \bar{\rho}_{p, q}(i, j)=1_{\{i=j\}}(p(i) \wedge q(i))+2 \frac{(p(i)-q(i))^{+}(q(j)-p(j))^{+}}{\|p-q\|} .
$$

One easily checks that $\sum_{i \neq j} \bar{\rho}_{p, q}(i, j)$ is equal to

$$
1-\sum_{i \in \overline{\mathbb{N}}}\left(p(i)-(p(i)-q(i))^{+}\right)=\sum_{i \in \overline{\mathbb{N}}}(p(i)-q(i))^{+}=\frac{1}{2}\|p-q\|=\inf _{\rho \in \mathcal{P}_{p, q}} \sum_{i \neq j} \rho(i, j) .
$$

We set $\tau$ equal to $T \wedge \tilde{T}$ under (SF) and equal to $T \wedge \tilde{T} \wedge \mathcal{T}_{p_{0}}$ otherwise. Let $\left(X_{t}, \tilde{X}_{t}\right)$ be a jump process on $\overline{\mathbb{N}}^{2}$ starting from $\left(X_{0}, \tilde{X}_{0}\right)$ distributed according to $\bar{\rho}_{p_{0}, \tilde{p}_{0}}$ and 
with transition rates $\lambda(t,(i, j),(k, l))$ equal to

$$
\left\{\begin{array}{l}
\tilde{F}_{k, i-k} \text { if } i=j \text { and } 1 \leq k=l<i \\
\left(\tilde{K}_{i, k-i} \wedge \tilde{K}_{i, l-i}\right) \bar{\rho}_{p_{t}, \tilde{p}_{t}}(k-i, l-i) \text { if } i=j \text { and } i<k, l<\infty \\
\sum_{m \in \mathbb{N}^{*}}\left(\tilde{K}_{i, k-i}-\tilde{K}_{i, m}\right)^{+} \bar{\rho}_{p_{t}, \tilde{p}_{t}}(k-i, m) \text { if } i=j=l \text { and } i<k<\infty \\
\sum_{m \in \mathbb{N}^{*}}\left(\tilde{K}_{i, l-i}-\tilde{K}_{i, m}\right)^{+} \bar{\rho}_{p_{t}, \tilde{p}_{t}}(m, l-i) \text { if } i=j=k \text { and } j<l<\infty \\
\lambda_{p}(t, i, k) \text { if } i \neq j \text { and } j=l \\
\lambda_{\tilde{p}}(t, j, l) \text { if } j \neq i \text { and } i=k
\end{array}\right.
$$

and 0 otherwise. In case of accumulation of jumps, the process behaves like the one introduced in the proof of Theorem 2.6. Since by Proposition 2.3 or Theorem 2.6, $\forall t \in[0, \tau), \sum_{i \in \mathbb{N}^{*}} p_{t}(i)=\sum_{i \in \mathbb{N}^{*}} \tilde{p}_{t}(i)=1$, one easily checks that $X_{t}$ (resp. $\left.\tilde{X}_{t}\right)$ is a jump process with transition rates $\lambda_{p}$ (resp. $\lambda_{\tilde{p}}$ ) starting from $X_{0}$ with law $p_{0}$ (resp. $\left.\tilde{p}_{0}\right)$ and according again to Proposition 2.3 or Theorem 2.6, for any $t \in[0, \tau)$, the law of $X_{t}$ (resp. $\tilde{X}_{t}$ ) is $p_{t}$ (resp. $\tilde{p}_{t}$ ). The coupling is constructed in order to minimize for any $i \in \mathbb{N}^{*}$ the total jump rate leading from $(i, i)$ to $(k, l)$ with $k \neq l$ :

$$
\sum_{k \neq l} \lambda(t,(i, i),(k, l))=\sum_{\substack{m, n \in \mathbb{N}^{*} \\ m \neq n}}\left(\tilde{K}_{i, m} \vee \tilde{K}_{i, n}\right) \bar{\rho}_{p_{t}, \tilde{p}_{t}}(m, n) \leq \frac{1}{2} \kappa i^{\gamma}\left\|p_{t}-\tilde{p}_{t}\right\|
$$

where $\gamma$ is equal to $\alpha$ under (SF) and to 1 otherwise. As the law of $\left(X_{t}, \tilde{X}_{t}\right)$ belongs to $\mathcal{P}_{p_{t}, \tilde{p}_{t}}$,

$$
\begin{aligned}
\left\|p_{t}-\tilde{p}_{t}\right\| & \leq 2 \mathbb{P}\left(X_{t} \neq \tilde{X}_{t}\right) \leq 2 \mathbb{P}\left(X_{0} \neq \tilde{X}_{0}\right)+2 \mathbb{P}\left(X_{0}=\tilde{X}_{0} \text { and } X_{t} \neq \tilde{X}_{t}\right) \\
& \leq\left\|p_{0}-\tilde{p}_{0}\right\|+2 \mathbb{P}\left(\exists s \leq t: X_{s^{-}}=\tilde{X}_{s^{-}} \text {and } X_{s} \neq \tilde{X}_{s}\right) \\
& \leq\left\|p_{0}-\tilde{p}_{0}\right\|+2 \sum_{i \in \mathbb{N}^{*}} \mathbb{E}\left(\sum_{s \leq t} 1_{\left\{X_{s^{-}}=\tilde{X}_{s^{-}}=i \text { and } X_{s} \neq \tilde{X}_{s}\right\}}\right) \\
& \leq\left\|p_{0}-\tilde{p}_{0}\right\|+\kappa \int_{0}^{t}\left\|p_{s}-\tilde{p}_{s}\right\| \sum_{i \in \mathbb{N}^{*}} i^{\gamma} \mathbb{P}\left(X_{s^{-}}=\tilde{X}_{s^{-}}=i\right) d s \\
& \leq\left\|p_{0}-\tilde{p}_{0}\right\|+\kappa \int_{0}^{t}\left\|p_{s}-\tilde{p}_{s}\right\| \sum_{i \in \mathbb{N}^{*}} i^{\gamma} \tilde{p}_{s}(i) d s
\end{aligned}
$$

where the penultimate inequality is a consequence of (3.1). The conclusion follows from Gronwall's Lemma combined with the estimate given in Lemma 2.4 under (SF) and with (2.5) otherwise.

REMARK 3.3. Being a little more cautious, one can prove by this approach [J] that there exists a unique couple $\left(\mathcal{T},\left(p_{t}\right)_{t \in[0, \mathcal{T})}\right)$ with $\mathcal{T} \geq \mathcal{T}_{p_{0}}$ and $p_{t}$ solution of (1.3) on $[0, \mathcal{T})$ such that $\sum_{i \in \mathbb{N}^{*}} i p_{t}(i)$ is locally integrable but not integrable on $[0, \mathcal{T})$. In addition uniqueness holds for $(1.3)$ on the time interval $[0, \mathcal{T})$. In case the coagulation kernel is subadditive: $K_{i, j} \leq \kappa(i+j), \mathcal{T}$ is equal to $+\infty$.

Acknowledgment. I thank Christophe Giraud for suggesting me to look at results concerning the dependence of solutions of (1.3) on the initial data rather than only considering uniqueness. 


\section{REFERENCES}

[BC] J.M. Ball and J. Carr, The discrete coagulation-fragmentation equations: Existence, uniqueness and density conservation, J. Statist. Phys., 61:203-234, 1990.

[D] F.P. Da Costa, Existence and uniqueness of density conserving solutions to the coagulationfragmentation equations with strong fragmentation, J. Math. Anal. Appl., 192:892-914, 1995.

[J] B. Jourdain, Nonlinear processes associated with the discrete Smoluchowski coagulationfragmentation equation, Markov Process. Related Fields, 9:103-130, 2003.

[SV] D. Stroock and S. Varadhan, Multidimensional Diffusion Processes, Springer-Verlag, 1997. 